in the vaccinia-related kinase 1 gene (VRK1). (Gonzaga-Jauregui C, Lotze T, Jamal L, et al. Mutations in VRK1 associated with complex motor and sensory axonal neuropathy plus microcephaly. JAMA Neurol 2013 Dec;70(12):1491-8).

COMMENTARY. Hereditary motor and sensory neuropathies (HMSNs) are a group of slowly progressive diseases genetically heterogeneous, with more than 40 disease-associated genes identified. VRK1 is a novel HMSN locus that can be associated with a complex peripheral neuropathy phenotype, an autosomal recessive axonal motor sensory neuropathy and microcephaly. Genome-wide analysis enables the identification of novel HMSN-associated genes.

\title{
AXONAL NEUROPATHY WITH NEUROMYOTONIA
}

Investigators from the Children's Hospital, Coimbra, Portugal, and centers in Belgium, report a 16-year-old girl with consanguineous parents who presented with progressive distal muscular atrophy and weakness, beginning at age 6 years. After 10 years follow-up, clinical myotonia developed and was confirmed by electrophysiologic studies. This severe chronic motor axonal neuropathy was associated with a homozygous mutation in HINT1 and with late onset neuromyotonia. Sensory impairment was discrete and also appeared late. (Caetano JS, Costa C, Baets J, et al. Autosomal recessive axonal neuropathy with neuromyotonia: A rare entity. Pediatr Neurol 2014 Jan;50(1):104-7).

COMMENTARY. Autosomal recessive axonal neuropathy with neuromyotonia is recently described in 50 patients from 33 families, with 8 different HINT1 mutations, in a report including the above patient and cited by the authors [1]. The disease presents in the first decade with distal muscle weakness in upper and lower limbs. Sensory impairment is mild in some and most develop action myotonia in the hands and orthopedic deformities.

\section{References.}

1. Zimon M, et al. Nat Genet. 2012 Oct;44(10):1080-3.

\section{LAMBERT-EATON SYNDROME IN CHILDREN}

Investigators from Boston Children's Hospital; the Lahey Clinic, Burlington, MA; and Ohio State University, report 3 children presenting between ages 9 and 10 years with proximal lower extremity weakness with areflexia and low-amplitude compound muscle action potentials, and diagnosed with Lambert-Eaton myasthenic syndrome. A literature review found 9 other pediatric cases of Lambert-Eaton myasthenic syndrome, 3 having associated malignancies, 2 with lymphoproliferative disorders and 1 with neuroblastoma. The 9 nonparaneoplastic patients with primary autoimmune disorder responded to immunomodulatory therapy, with complete remission in 2 patients. (Hajjar M, Markowitz J, Darras BT, Kissel JT, Srinivasan J, Jones HR. Lambert-Eaton syndrome, an unrecognized treatable pediatric neuromuscular disorder: Three patients and literature review. Pediatr Neurol 2014 Jan;50(1):11-7). 